\title{
NEURAL NETWORK CONTROL OF \\ DISCRETE-TIME NON-AFFINE MIMO SYSTEMS WITH DISTURBANCES
}

\author{
Jin Zhang ${ }^{*, 1}$ Shuling Dai * \\ * The Advanced Simulation Technology Laboratory \\ School of Automation \\ Beijing University of Aeronautics and Astronautics
}

\begin{abstract}
In this paper, a simple neural network (NN) control scheme is developed for a class of discrete-time multi-input multi-output (MIMO) non-affine nonlinear systems with triangular form inputs and disturbances. The system studied is described by NARMAX (Nonlinear Auto Regressive Moving Average with eXogenous inputs) model. Firstly, by using implicit function theorem, the existence of the implicit desired feedback control (IDFC) is proved. Then single layer neural networks are used as the emulators of the desired controls. The stability of the closed-loop system is rigorously proved by using Lyapunov method. Because only input and output sequences are needed to construct the approximation based controls, the method proposed is very simple to be implemented in practical applications. Copyright (C)2005 IFAC
\end{abstract}

Keywords: MIMO system, neural networks, discrete-time system.

\section{INTRODUCTION}

Neural networks control of nonlinear systems has been extensively studied in the past decades. The universal approximation ability of neural networks makes it one of the effective tool in nonlinear system identification and control(Cabrera and Narendra, 1999; Narendra and Parthasarathy, 1990; Polycarpou, 1996). Most often used neural networks include Radial Basis Function (RBF) neural networks (Lewis et al., 1999; Ge et al., 2001), HONNs (Kosmatopoulos et al., 1995) and Multi-layer Neural Networks (MNNs) (Lewis et al., 1999; Ge et al., 2001). In this paper, single layer neural networks (RBF and HONN) are used to approximate the unknown nonlinear implicit desired feedback controls, which can drive the

\footnotetext{
1 Corresponding author. Tel. 86-10-82316226; Fax. 86-1082316226; E-mail: jinzhang2004@hotmail.com
}

outputs of the MIMO systems studied to track the desired trajectories.

Nowadays, because most of the control systems are implemented in digital computers, the study on discrete-time systems are attracting more and more attentions. One of the most popular nonlinear discrete-time systems studied in the literature is the NARMAX systems (Leontaritis and Billings, 1985), in which, only input output data are used in the description of the system model. Due to most of the discrete time systems in state space representation can be transformed into the NARMAX description (Ge et al., 2003d; Zhang et al., 2003), the study on control and identification of NARMAX models by output feedback is meaningful. SISO NARMAX systems have been extensively studied in (Billings and Voon, 1986; Chen and Khalil, 1995; Ge et al., 2003d; Ge et al., May, 2003b). For MIMO NARMAX models, relative less results have been obtained. Previous works 
on MIMO NARMAX systems include (Ge et al., 2003c; Jagannathan and Lewis, 1996a; Jagannathan and Lewis, 1996b; Sun et al., 1998). Unfortunately, all of those works deal with affine systems, i.e., the control inputs appear linearly, which makes the feedback linearization method applicable. For MIMO non-affine systems, even fewer results have been obtained.

In (Ge et al., 2003c), neural network control scheme was investigated for a class of MIMO NARMAX discrete time systems. The $\tau$-step weight update laws was proved to be effective in handling the $\tau$-step predictor model in the presence of unknown bounded disturbances. However, the system studied is in affine form and an orthogonal matrix should be found in order to update the NN weights. In this paper, the system studied is in non-affine MIMO NARMAX form. For the $n \times n$ MIMO systems, the inputs of the system are in triangular form. Due to this property and by implicit function theorem (Ge et al., 2001), we can firstly define the IDFC controls in a nested manner, then using neural networks to emulate those IDFC. The main contributions of this paper can be summarized as follows: (i) An effective NN control scheme is developed for a class of nonaffine nonlinear discrete-time MIMO systems with triangular form inputs; (ii) The proposed method is very simple for practical implementation; (iii) The closed loop system is proved to be stable in the presence of bounded disturbances.

This paper is organized as follows. System dynamics as well as some stability notions are proposed in Section 2. Controller design and stability analysis are discussed in Section 3. Finally, conclusions are made in Section 4. Due to space limitation, simulation results are omitted in this paper.

\section{MIMO SYSTEM DYNAMICS}

Considering the following $n \times n$ non-affine nonlinear NARMAX MIMO systems with triangular form inputs

$$
\left\{\begin{aligned}
y_{1}(k+\tau)= & f_{1}\left(Y(k), U_{k-1}(k), D_{k-1}(k),\right. \\
& \left.D_{k+\tau-1}(k), u_{1}(k)\right) \\
\vdots & \\
y_{j}(k+\tau)= & f_{j}\left(Y(k), U_{k-1}(k), D_{k-1}(k),\right. \\
& \left.D_{k+\tau-1}(k), u_{1}(k), \ldots, u_{j}(k)\right) \\
\vdots & \\
y_{n}(k+\tau)= & f_{n}\left(Y(k), U_{k-1}(k), D_{k-1}(k),\right. \\
& \left.D_{k+\tau-1}(k), u_{1}(k), \ldots, u_{n}(k)\right)
\end{aligned}\right.
$$

where $\tau$ is the system delay; $y(k)=\left[y_{1}(k), \ldots\right.$, $\left.y_{n}(k)\right]^{T} \in R^{n}$ and $u(k)=\left[u_{1}(k), \ldots, u_{n}(k)\right]^{T} \in$ $R^{n}$ are system outputs and inputs, respectively;
$Y(k)$ is a vector containing current and past outputs, $U_{k-1}(k)$ is a vector containing only past inputs; $D_{k-1}(k)$ and $D_{k+\tau-1}(k)$ represent the past and future (include current disturbance) bounded disturbances respectively. In particular, they are defined as

$$
\begin{aligned}
U_{k-1}(k)= & {\left[u_{1}(k-1), \ldots, u_{1}\left(k-m_{1}\right),\right.} \\
& u_{2}(k-1), \ldots, u_{2}\left(k-m_{2}\right), \\
& \ldots, \\
& \left.u_{n}(k-1), \ldots, u_{n}\left(k-m_{n}\right)\right]^{T} \\
Y(k)= & {\left[y_{1}(k), \ldots, y_{1}\left(k-n_{1}+1\right),\right.} \\
& y_{2}(k), \ldots, y_{2}\left(k-n_{2}+1\right), \\
& \ldots, \\
& \left.y_{n}(k), \ldots, y_{n}\left(k-n_{n}+1\right)\right]^{T} \\
D_{k-1}(k)= & {[d(k-1), \ldots, d(k-h)]^{T} } \\
D_{k+\tau-1}(k)= & {[d(k+\tau-1), d(k+\tau-2), \ldots, d(k)]^{T} }
\end{aligned}
$$

with $n_{i}$ denotes the length of the $i$-th subsystem's outputs, and $m_{i}$ is the length of the $i$-th subsystem's inputs, which satisfies $m_{i}<n_{i}, i=$ $1, \ldots, n ; h$ denotes the length of the past disturbances; $f_{j}(\cdot)$ are nonlinear functions; $\bar{u}_{j-1}(k)=$ $\left.\left[u_{1}(k), \ldots, u_{j-1}(k)\right)\right]^{T}$. For the ease of analysis, define $(j=1, \ldots, n)$

$$
\begin{array}{r}
f_{j}\left(k, u_{1}(k), \ldots, u_{j}(k)\right) \triangleq \\
f_{j}\left(Y(k), U_{k-1}(k), D_{k-1}(k), D_{k+\tau-1}(k),\right. \\
\left.u_{1}(k), \ldots, u_{j}(k)\right)
\end{array}
$$

The control objective is to design control input $u(k)$ for system (1) to drive the system output $y(k)$ follow a known and bounded trajectory $y_{d}(k)=\left[y_{d_{1}}(k), y_{d_{2}}(k), \ldots, y_{d_{n}}(k)\right]^{T} \in R^{n}$.

Assumption 2.1. The desired trajectory $y_{d}(k) \in$ $\Omega_{y d} \subset R^{p}, \quad \forall k>0$ is smooth and known, where $\Omega_{y d}$ is a small subset of $\Omega_{y}$ and $\Omega_{y} \triangleq$ $\{\chi(k) \mid \chi(k)=y(k)\} \subset R^{p}$.

Assumption 2.2. There are positive constants $\underline{\mu}_{i}$ and $\bar{\mu}_{i}(i=1, \ldots, n)$, such that $0<\underline{\mu}_{i} \stackrel{\underline{\mu}_{i}}{\leq}$ $\left|\frac{\partial f_{i}\left(k, u_{1}(k), \ldots, u_{i}(k)\right)}{\partial u_{i}}\right| \leq \bar{\mu}_{i}$.

Remark 2.1. The partial derivative $\frac{\partial f_{i}(\cdot)}{\partial u_{i}(k)}$ can be considered as the controller gain of the $i$-th input for the $i$-th subsystem. Assumption 2.2 indicates that this control gain is either positive or negative, and is also upper and lower bounded. The sign does not need to be known a priori.

Assumption 2.3. The function $f_{i}\left(k, u_{1}(k), \ldots, u_{i}(k)\right)$ is locally Lipschitz in $D_{k-1}(k)$ and $D_{k+\tau-1}(k)$ at $(0,0)$, i.e., there are Lipschitz constants $L_{1}$ and $L_{2}$ such that 


$$
\begin{gathered}
\| f_{i}\left(Y(k), U_{k-1}(k), D_{k-1}(k), D_{k+\tau-1}(k),\right. \\
\left.u_{1}(k), \ldots, u_{i}(k)\right) \\
-f_{i}\left(Y(k), U_{k-1}(k), 0,0, u_{1}(k), \ldots, u_{i}(k)\right) \| \\
\leq L_{1}\left\|D_{k-1}(k)\right\|+L_{2}\left\|D_{k+\tau-1}(k)\right\|
\end{gathered}
$$

Assumption 2.4. The disturbances vector $D_{k-1}(k)$ and $D_{k+\tau-1}(k)$ are bounded, i.e., $\left\|D_{k-1}(k)\right\| \leq$ $D_{1}$ and $\left\|D_{k+\tau-1}(k)\right\| \leq D_{2}$ with $D_{1}$ and $D_{2}$ are positive constants.

Assumption 2.5. The nonlinear functions $f_{i}(k$, $\left.u_{1}(k), \ldots, u_{i}(k)\right)(i=1, \ldots, n)$ are differentiable.

In the following, Lemma 2.1 (Mean Value Theorem for multi variables), Lemma 2.2 (Implicit Function Theorem) and Lemma 2.3 (Bounded Input Bounded Output) are given, which will be used later.

Lemma 2.1. Let $f: R^{n} \rightarrow R$ be differentiable at every point in an open set containing the line segment $L$ joining two vectors $\bar{a}$ and $\bar{b}$ in $R^{n}$, then there is a vector $\bar{\xi}$ on $L$ such that

$$
f(\bar{b})-f(\bar{a})=\nabla f(\bar{\xi}) \cdot(\bar{b}-\bar{a})
$$

with $\nabla f(\cdot)$ denotes the gradient of $f(\cdot)$.

Lemma 2.2. Assume that $f(x, y): R^{n} \times R \rightarrow R$ is continuously differentiable $\forall(x, y) \in R^{n} \times R$, and there exists a positive constant $d$ such that $\partial f(x, y) / \partial y(x, y)>d>0, \forall(x, y) \in R^{n} \times R$. Then there exists a continuous (smooth) function $y^{*}=g(x)$ such that $f\left(x, y^{*}\right)=0$. For the case $\partial f(x, y) / \partial y(x, y)<-d<0, \forall(x, y) \in R^{n} \times R$. The result still holds (Ge et al., 2001).

Lemma 2.3. : Consider the linear time varying discrete-time system given by

$$
x(k+1)=A(k) x(k)+B u(k), y(k)=C x(k)(2)
$$

where $A(k), B$ and $C$ are appropriately dimensional matrices with $B$ and $C$ are constant matrices. Let $\Phi\left(k_{1}, k_{0}\right)$ be the state-transition matrix corresponding to $A(k)$ for system (2), i.e. $\Phi\left(k_{1}, k_{0}\right)=\prod_{k=k_{0}}^{k_{1}-1} A(k)$. If $\left\|\Phi\left(k_{1}, k_{0}\right)\right\|<1, \forall k_{1}>$ $k_{0} \geq 0$, then system (2) is (i) globally exponentially stable for the unforced system (i.e. $u(k)=0$ ); and (ii) bounded-input-boundedoutput (BIBO) stable (Ge et al., 2003d).

Define the tracking error $e(k)=y(k)-y_{d}(k)$ as

$$
\begin{aligned}
e(k) & =\left[e_{1}(k), \ldots, e_{n}(k)\right]^{T} \\
& =\left[y_{1}(k)-y_{d_{1}}(k), \ldots, y_{n}(k)-y_{d_{n}}(k)\right]^{T}(3)
\end{aligned}
$$

Considering the first equation in (1), subtracting $y_{d_{1}}(k+\tau)$ on both sides, we have

$$
\begin{aligned}
e_{1}(k+\tau)= & f_{1}\left(Y(k), U_{k-1}(k), 0,0, u_{1}(k)\right) \\
& -y_{d_{1}}(k+\tau)+\Delta_{1}(k)
\end{aligned}
$$

with $\Delta_{1}(k) \triangleq f_{1}\left(k, u_{1}(k)\right)-f_{1}\left(Y(k), U_{k-1}(k), 0\right.$, $\left.0, u_{1}(k)\right)$.

Noting Assumption 2.2, we can obtain

$$
\left|\frac{\partial\left[f_{1}\left(Y(k), U_{k-1}(k), 0,0, u_{1}(k)\right)-y_{d_{1}}(k+\tau)\right]}{\partial u_{1}}\right|
$$

by Lemma 2.2 , we know that there is

$$
\begin{aligned}
u_{1}^{*}(k) & \triangleq \alpha_{1}^{\prime}\left(Y(k), U_{k-1}(k), y_{d_{1}}(k+\tau)\right) \\
& \triangleq \alpha_{1}\left(Y(k), U_{k-1}(k), y_{d}(k+\tau)\right)
\end{aligned}
$$

such that $f_{1}\left(Y(k), U_{k-1}(k), 0,0, u_{1}(k)\right)-y_{d_{1}}(k+$ $\tau)=0$, therefore we have

$$
\begin{aligned}
e_{1}(k+\tau)= & f_{1}\left(Y(k), U_{k-1}(k), 0,0, u_{1}^{*}(k)\right) \\
& -y_{d_{1}}(k+\tau)+\Delta_{1}(k) \\
= & \Delta_{1}(k)
\end{aligned}
$$

By assumption 2.3 and 2.4, we know that $\Delta_{1}(k)$ is bounded. That is to say, the tracking error is bounded. Furthermore, if there are no disturbances, we can obtain $\Delta_{1}(k)=0$, i.e, exact tracking can be achieved.

Remark 2.2. It should be noted that though $u_{1}^{*}(k)$ only depends on $Y(k), U_{k-1}(k)$ and $y_{d_{1}}(k+\tau)$, for the ease of analysis, we regard it as a function of $Y(k), U_{k-1}(k)$ and $y_{d}(k+\tau)$.

Once $u_{1}(k)$ is fixed as $u_{1}^{*}(k)$, by following the same procedure and considering the second equation in (1), we have

$$
\begin{aligned}
e_{2}(k+\tau)= & f_{2}\left(Y(k), U_{k-1}(k), 0,0, u_{1}^{*}(k), u_{2}(k)\right) \\
& -y_{d_{2}}(k+\tau)+\Delta_{2}(k)
\end{aligned}
$$

with $\Delta_{2}(k) \triangleq f_{2}\left(k, u_{1}(k), u_{2}(k)\right)-f_{2}(Y(k)$, $\left.U_{k-1}(k), 0,0, u_{1}(k), u_{2}(k)\right)$. Choosing $u_{1}(k)=$ $u_{1}^{*}(k)=\alpha_{1}\left(Y(k), U_{k-1}(k), y_{d}(k+\tau)\right)$, noting Assumption 2.5 and by Lemma 2.2, we know that there is an ideal control

$$
\begin{aligned}
u_{2}^{*}(k) & \triangleq \alpha_{2}^{\prime}\left(Y(k), U_{k-1}(k), y_{d_{1}}(k+\tau), y_{d_{2}}(k+\tau)\right) \\
& \triangleq \alpha_{2}\left(Y(k), U_{k-1}(k), y_{d}(k+\tau)\right)
\end{aligned}
$$

such that $f_{2}\left(Y(k), U_{k-1}(k), 0,0, u_{1}^{*}(k), u_{2}(k)\right)-$ $y_{d_{2}}(k+\tau)=0$, therefore we have

$$
\begin{aligned}
e_{2}(k+\tau)= & f_{2}\left(Y(k), U_{k-1}(k), 0,0, u_{1}^{*}(k), u_{2}^{*}(k)\right) \\
& -y_{d_{2}}(k+\tau)+\Delta_{2}(k) \\
= & \Delta_{2}(k)
\end{aligned}
$$

Similarly, we know that there are ideal controls 


$$
\begin{aligned}
u_{3}^{*}(k) & \triangleq \alpha_{3}\left(Y(k), U_{k-1}(k), y_{d}(k+\tau)\right) \\
& \cdots \\
u_{j}^{*}(k) & \triangleq \alpha_{j}\left(Y(k), U_{k-1}(k), y_{d}(k+\tau)\right) \\
& \cdots \\
u_{n}^{*}(k) & \triangleq \alpha_{n}\left(Y(k), U_{k-1}(k), y_{d}(k+\tau)\right)
\end{aligned}
$$

such that

$$
\left\{\begin{aligned}
e_{3}(k+\tau) & =\Delta_{3}(k) \\
& \vdots \\
e_{j}(k+\tau) & =\Delta_{j}(k) \\
& \vdots \\
e_{n}(k+\tau) & =\Delta_{n}(k)
\end{aligned}\right.
$$

Defination 2.1. The ideal controls $u_{1}^{*}(k), u_{2}^{*}(k)$, $\ldots, u_{n}^{*}(k)$, which can realize exact tracking in $\tau$ steps and cannot be explicitly spelt out, are called implicit desired feedback control (IDFC).

Summarizing equations (4), (5) and (6), we can see that the $i$-th IDFC, $u_{i}^{*}(k)$, can be expressed as follows

$$
u_{i}^{*}(k)=\alpha_{i}(z(k)), \quad i=1,2, \ldots, n
$$

with $z(k) \triangleq\left[Y^{T}(k), U_{k-1}^{T}(k), y_{d}^{T}(k+\tau)\right]^{T} \in$ $R^{\sum_{j=1}^{n}\left(m_{j}+n_{j}\right)+n}$. Its vector form is as follows

$$
u^{*}(k)=\left[\begin{array}{c}
\alpha_{1}(z(k)) \\
\alpha_{2}(z(k)) \\
\vdots \\
\alpha_{n}(z(k))
\end{array}\right] \in R^{n \times 1}
$$

It can be seen that system (1) is in non-affine form. For the convenience of analysis, denote system (1) in the following vector form

$$
\begin{aligned}
y(k+\tau)= & F\left(Y(k), U_{k-1}(k),\right. \\
& \left.D_{k-1}(k), D_{k+\tau-1}(k), u(k)\right)
\end{aligned}
$$

with nonlinear vector function $F(\cdot) \in R^{n \times 1}$ is defined as

$$
F(\cdot)=\left[\begin{array}{l}
f_{1}\left(k, u_{1}(k)\right) \\
\vdots \\
f_{j}\left(k, u_{1}(k), \ldots, u_{j}(k)\right) \\
\vdots \\
f_{n}\left(k, u_{1}(k), \ldots, u_{n}(k)\right)
\end{array}\right]
$$

Therefore, we have

$$
\begin{aligned}
e(k+\tau)= & F\left(Y(k), U_{k-1}(k), D_{k-1}(k),\right. \\
& \left.D_{k+\tau-1}(k), u(k)\right)-y_{d}(k+\tau)(10)
\end{aligned}
$$

Adding and subtracting $F\left(Y(k), U_{k-1}(k), 0,0\right.$, $\left.u^{*}(k)\right)$ to the right side of equation (10), we have

$$
\begin{aligned}
& e(k+\tau)= \\
& \quad F\left(Y(k), U_{k-1}(k), D_{k-1}(k), D_{k+\tau-1}(k), u(k)\right) \\
& \quad-F\left(Y(k), U_{k-1}(k), 0,0, u^{*}(k)\right)
\end{aligned}
$$

Considering the $i$-th $1 \leq i \leq n$ equation in the error dynamics (11), adding and subtracting $f_{i}\left(Y(k), U_{k-1}(k), D_{k-1}(k), D_{k+\tau-1}(k), u_{1}^{*}(k)\right.$, $\left.\ldots, u_{i}^{*}(k)\right)$ to the right side of the $i$-th error equation, we have

$$
\begin{aligned}
e_{i}(k+\tau)= & f_{i}\left(k, u_{1}(k), \ldots, u_{i}(k)\right) \\
& -f_{i}\left(k, u_{1}^{*}(k), \ldots, u_{i}^{*}(k)\right)+\Delta_{i}^{*}(k)
\end{aligned}
$$

with $\Delta_{i}^{*}(k) \triangleq f_{i}\left(Y(k), U_{k-1}(k), D_{k-1}(k), D_{k+\tau-1}(k)\right.$, $\left.u_{1}^{*}(k), \ldots, u_{i}^{*}(k)\right)-f_{i}\left(Y(k), U_{k-1}(k), 0,0, u_{1}^{*}(k)\right.$, ..., $\left.u_{i}^{*}(k)\right)$. By assumption 2.3 and 2.4, we can obtain that $\Delta_{i}^{*}(k)(i=1, \ldots, n)$ is bounded.

By noting Lemma 2.1, the Mean Value Theorem for multi variables, equation (12) can be written as

$$
\begin{aligned}
e_{i}(k+\tau)= & \nabla f_{i}\left(k, \bar{u}_{\xi_{i}}\right)\left[\bar{u}_{i}(k)-\bar{u}_{i}^{*}(k)\right] \\
& +\Delta_{i}^{*}(k)
\end{aligned}
$$

with

$$
\begin{aligned}
\bar{u}_{\xi_{i}} & =\left[u_{\xi_{i 1}}, u_{\xi_{i 2}}, \ldots, u_{\xi_{i i}}\right]^{T} \\
\nabla f_{i}\left(k, u_{\xi_{i}}\right) & =\left[\frac{\partial f_{i}}{\partial u_{1}}, \frac{\partial f_{i}}{\partial u_{2}}, \ldots, \frac{\partial f_{i}}{\partial u_{i}}\right] \in R^{1 \times i}
\end{aligned}
$$

with $\bar{u}_{\xi_{i}} \in\left[\bar{u}_{i}^{*}(k), \bar{u}_{i}(k)\right]$.

Then equation (11) can be written as

$$
e(k+\tau)=\nabla F(k) \cdot\left[u(k)-u^{*}(k)\right]+\Delta^{*}(k)(14)
$$

with $\Delta^{*}(k) \triangleq\left[\Delta_{1}^{*}(k), \ldots, \Delta_{n}^{*}(k)\right]^{T}$ and

$$
\begin{aligned}
& \nabla F(k) \triangleq\left[\nabla f_{1}\left(k, u_{\xi_{1}}\right), \ldots, \nabla f_{n}\left(k, u_{\xi_{n}}\right)\right]^{T} \\
& =\left[\begin{array}{ccc}
\left.\frac{\partial f_{1}}{\partial u_{1}}\right|_{u_{1}(k)=u_{\xi_{11}}} & 0 & 0 \\
\vdots & \ddots & 0 \\
\left.\frac{\partial f_{n}}{\partial u_{1}}\right|_{u_{1}(k)=u_{\xi_{n 1}}} & \cdots & \left.\frac{\partial f_{n}}{\partial u_{n}}\right|_{u_{n}(k)=u_{\xi_{n n}}}
\end{array}\right]
\end{aligned}
$$

For the ease of analysis, define

$$
G(k) \triangleq \nabla F(k)
$$

Therefore, we have

$$
e(k+\tau)=G(k)\left[u(k)-u^{*}(k)\right]+\Delta^{*}(k)
$$

It can be easily obtained that the matrix $G(k)$ possess the following properties:

(1) $|G(k)|=\Pi_{i=1}^{n}\left(\left.\frac{\partial f_{i}}{\partial u_{i}}\right|_{u_{i}(k)=u_{\xi_{i i}}}\right) \neq 0$; 
(2) $G(k)$ is upper and lower bounded, i.e, there are two constants $a=\Pi_{i=1}^{n} \mu_{i}$ and $b=$ $\Pi_{i=1}^{n} \bar{\mu}_{i}$, such that $a I \leq G(k) \leq \bar{b} I(a, b>0)$ or $b I \leq G(k) \leq a I(a, b<0)$.

It can be seen that the matrix $G(k)$ is either positive or negative, which depends on the signs of its diagonal elements. In the following, without losing of generality, we assume that $G(k)$ is positive, i.e, $a I \leq G(k) \leq b I(a, b>0)$. Therefore, we can obtain

$$
\frac{1}{b} I \leq G^{-1}(k) \leq \frac{1}{a} I, \quad a, b>0
$$

\section{STABILITY ANALYSIS}

Considering the IDFCs defined in equation (8), they are continuous nonlinear functions. Therefore, there are ideal weights $W^{*}$ such that the smooth function vector $u^{*}(k)$ can be approximated by an ideal NN on a compact set $\Omega_{z} \subset R^{q}$

$$
u^{*}(k)=W^{* T} S(z(k))+\epsilon_{z}
$$

where $z(k)$ has been defined in equation (7) as follows

$$
\begin{aligned}
z(k)= & {\left[\begin{array}{c}
Y(k) \\
U_{k-1}(k) \\
y_{d}(k+\tau)
\end{array}\right] \in \Omega_{z} \subset R^{q} } \\
& q=\sum_{i=1}^{n}\left(n_{i}+m_{i}\right)+n \\
\epsilon_{z}= & {\left[\epsilon_{z_{1}}, \ldots, \epsilon_{z_{n}}\right]^{T} }
\end{aligned}
$$

and $\epsilon_{z}$ is the bounded NN approximation error vector satisfying $\left\|\epsilon_{z}\right\| \leq \epsilon_{0}\left(\epsilon_{0}\right.$ is a constant vector) on the compact set, which can be reduced by increasing the number of the adjustable weights. The ideal weight matrix $W^{*}$ is required for analytical purpose only, and is defined as that minimizes $\left\|\epsilon_{z}\right\|$ for all $z(k) \in \Omega_{z} \subset R^{q}$ in a compact region, i.e.,

$W^{*} \triangleq \arg \min _{W \in \Omega_{w}}\left\{\sup _{z \in \Omega_{z}}\left|u^{*}(k)-W^{T} S(z(k))\right|\right\}$

with $\Omega_{z} \subset R^{q}$ and compact set $\Omega_{w} \subset R^{l \times p}$. In general, the ideal $\mathrm{NN}$ weight matrix, $W^{*}$, is unknown though constant, its estimate, $\hat{W}$, should be used for controller design which will be discussed in the following.

Choosing the practical neural network controls and corresponding weight update laws as follows

$$
\begin{aligned}
u(k)= & \hat{W}^{T}(k) S(z(k)) \\
\hat{W}(k+1)= & \hat{W}(k-\tau+1) \\
& -\Gamma\left[S(z(k-\tau+1)) e^{T}(k+1)\right. \\
& +\sigma \hat{W}(k-\tau+1)]
\end{aligned}
$$

where $\Gamma=\gamma I$ with $\gamma>0, \sigma$ is a positive constant number and assume $0<1-\sigma \gamma<1, \hat{W}(k) \in R^{p \times l}$ and $S(z(k)) \in R^{l}$. For the ease of analysis, we rewrite equation (22) as follows

$$
\begin{aligned}
& \hat{W}(k+\tau)= \\
& \hat{W}(k)-\Gamma\left[S(z(k)) e^{T}(k+\tau)+\sigma \hat{W}(k)\right](23)
\end{aligned}
$$

Noting equation (17), we can obtain that

$$
\begin{aligned}
e(k+\tau)= & G(k)\left[\hat{W}^{T}(k) S(z(k))-W^{*^{T}}(k) S(z(k))\right. \\
& \left.-\epsilon_{z}\right]+\Delta^{*}(k)
\end{aligned}
$$

Thus, we can obtain

$$
\tilde{W}^{T}(k) S(z(k))=G^{-1}(k) e(k+\tau)+\epsilon_{d}
$$

with $\epsilon_{d}=\epsilon_{z}-G^{-1}(k) \Delta^{*}(k)$ is bounded.

Theorem 3.1. Consider the closed-loop system consisting of system (1), controller (21) and adaptation law (22). There exist compact sets $\Omega_{y_{0}} \subset$ $\Omega_{y}, \Omega_{w_{0}} \subset \Omega_{w}$ and positive constants $l^{*}, \gamma^{*}$ and $\sigma^{*}$ such that if

(1) All the assumptions being satisfied, the condition at time instant $k_{0}$ is initialized as

$$
\begin{aligned}
& y\left(k_{0}-j\right) \in \Omega_{y_{0}}, \\
& \quad j=0, \ldots, \max \left\{n_{1}, \ldots, n_{n}\right\}-1 \\
& u\left(k_{0}-j\right) \in \Omega_{u}, \\
& \quad j=1, \ldots, \tau+\max \left\{m_{1}, \ldots, m_{n}\right\} \\
& \tilde{W}\left(k_{0}-j\right) \in \Omega_{w_{0}}, \\
& \quad j=0, \ldots, \tau-1
\end{aligned}
$$

(2) the semi determined future outputs at time instant $k_{0}, y\left(k_{0}+1\right), \ldots, y\left(k_{0}+\tau-1\right)$ are all in compact set $\Omega_{y}$, and

(3) the design parameters are suitably chosen such that $l>l^{*}, \sigma<\sigma^{*}$ and $\gamma<\gamma^{*}$ with $\gamma$ being the eigenvalue of $\Gamma$,

then, the closed-loop system is SGUUB.

Proof: Choose Lyapunov function candidate as

$$
\begin{aligned}
J(k)= & \frac{1}{b} \sum_{j=0}^{\tau-1} \operatorname{tr}\left\{e(k+j) e^{T}(k+j)\right\} \\
& +\sum_{j=0}^{\tau-1} \operatorname{tr}\left\{\tilde{W}^{T}(k+j) \Gamma^{-1} \tilde{W}(k+j)\right\}(25)
\end{aligned}
$$

where $b$ is the positive constant, which denotes the upper bound of the matrix $G(k)$. Detailed proof procedure can be done by following the proof procedure in (Ge et al., 9-12 Dec. 2003a), due to space limitation, it is omitted here.

We have

$$
\Delta J(k) \leq-\frac{1}{b} e^{T}(k+\tau)\{1-2 \sigma \gamma
$$




$$
\begin{aligned}
& -\gamma b(1+\sigma+l)\} e(k+\tau)-b e^{T}(k) e(k) \\
& +C_{0}
\end{aligned}
$$

with $C_{0}=\sigma\left\|W^{*}\right\|_{F}^{2}+\sigma \gamma \alpha_{0}^{T} \alpha_{0}+\frac{1}{\gamma} \epsilon_{d}^{T} \epsilon_{d}$ being a positive constant. If we choose the design parameters as follows

$$
\frac{1}{\gamma}>2 \sigma+b(1+\sigma+l)
$$

then we can obtain

$$
\Delta J(k) \leq-b e^{T}(k) e(k)+C_{0}
$$

then $\Delta J(k) \leq 0$ once any of the tracking errors $\left|e_{i}(k)\right|, i=1, \ldots, p$ is larger than $\sqrt{\frac{C_{0}}{b}}$. Furthermore, the tracking error $e(k)$ will converge to the compact set denoted by

$$
\Omega_{e_{0}} \triangleq\left\{e(k)|| e_{i}(k) \mid \leq \sqrt{\frac{C_{0}}{b}}, i=1, \ldots, p\right\}
$$

Due to negativeness of $\Delta J(k)$, we conclude that $e(k+\tau)$ must converges to the compact set $\Omega_{e_{0}}$ if $e(k)$ outside of $\Omega_{e_{0}}$ and all other conditions hold. Thus $y(k+\tau) \in \Omega_{y}$ will still hold if $\Omega_{e_{0}} \subset \Omega_{e}$.

\section{CONCLUSION}

In this paper, a simple NN control scheme was developed for a class of discrete time nonlinear non-affine MIMO systems with disturbances. The inputs of the MIMO system are in triangular form. By using implicit function theorem, firstly, the existence of the IDFCs was shown. Then single layer neural networks were used as the emulators of the IDFCs. Only input and output sequences were used to construct the effective neural network control, which is simple for practical implementation. Finally, the closed-loop system was proved to be SGUUB based on Lyapunov analysis.

\section{REFERENCES}

Billings, S. A. and W. S. F. Voon (1986). A prediction-error and stepwise regression algorithm for nonlinear systems. Internatonal Journal of Control 44, 803-822.

Cabrera, João. B. D. and Kumpati. S. Narendra (1999). Issues in the application of neural networks for tracking based on inverse control. IEEE Transaction on Automatic Control 44(11), 2007-2027.

Chen, F. C. and H. K. Khalil (1995). Adaptive control of a class of nonlinear discrete-time systems using neural networks. IEEE Transactions on Automatic Control 72(7), 791807.
Ge, S. S., C. C. Hang, T. H. Lee and T. Zhang (2001). Stable Adaptive Neural Network Control. Kluwer Academic. Boston, MA.

Ge, S. S., G. Y. Li and N. Xi (9-12 Dec. 2003a). Direct adaptive control for a class of multiinput and multi-output nonlinear systems using neural networks. Proceedings of the 42nd IEEE Conference on Decision and Control.

Ge, S. S., G. Y. Li and T. H. Lee (May, 2003b). Adaptive NN control for a class of strictfeedback discrete-time nonlinear systems. $A u$ tomatica 39(5), 807-819.

Ge, S. S., G. Y. Li, J. Zhang and T. H. Lee (2003c). Direct adaptive control for a class of MIMO nonlinear systems using neural networks. accepted by IEEE Transactions on Automatic Control.

Ge, S. S., T. H. Lee, G. Y. Li and J. Zhang $(2003 d)$. Adaptive NN control for a class of discrete-time nonlinear systems. International Journal of Control 76(4), 334-354.

Jagannathan, S. and F. L. Lewis (1996a). Discrete-time neural net controller for a class of nonlinear dynamical systems. IEEE Transaction on Automatic Control 41(11), 16931699.

Jagannathan, S. and F. L. Lewis (1996b). Multilayer discrete-time neural-net controller with guaranteed performance. IEEE Trans. Neural Network 7(1), 107-130.

Kosmatopoulos, E. B., M. M. Polycarpou, M. A. Christodoulou and P. A. Ioannou (1995). High-order neural network structures for identification of dynamical systems. IEEE Trans. Neural Networks 6(2), 422-431.

Leontaritis, I. J. and S. A. Billings (1985). Input-output parametric models for nonlinear systems. International Journal of Control 41(2), 303-344.

Lewis, F. L., S. Jagannathan and A. Yesildirek (1999). Neural Network Control of Robot Manipulators and Nonlinear Systems. London: Taylor \& Francis.

Narendra, K. S. and K. Parthasarathy (1990). Identification and control of dynamic systems using neural networks. IEEE Transactions on Neural Networks 1(1), 4-27.

Polycarpou, M. M. (1996). Stable adaptive neural control scheme for nonlinear systems. IEEE Transactions on Automatic Control 41(3), 447-451.

Sun, F., Z. Sun and P. Y. Woo (1998). Stable neural-network-based adaptive control for sampled-data nonlinear systems. IEEE Trans. Neural networks 9(5), 956-968.

Zhang, J., S. S. Ge and T. H. Lee (2003). Output feedback control of a class of discrete mimo nonlinear systems with triangular form inputs. submitted to IEEE Transactions on Neural Networks. 